\title{
Low-level laser therapy induces an upregulation of collagen gene expression during the initial process of bone healing: a microarray analysis
}

Carla Roberta Tim

Paulo Sérgio Bossini Hueliton Wilian Kido Iran Malavazi

Marcia Regina von Zeska Kress

Marcelo Falsarella Carazzolle

Ana Cláudia Rennó

Nivaldo Antonio Parizotto 


\title{
Low-level laser therapy induces an upregulation of collagen gene expression during the initial process of bone healing: a microarray analysis
}

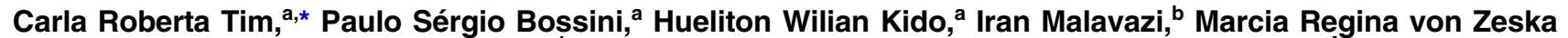 \\ Kress, ${ }^{c}$ Marcelo Falsarella Carazzolle, ${ }^{d, e}$ Ana Cláudia Rennó, ${ }^{a}$ and Nivaldo Antonio Parizotto ${ }^{f}$ \\ ${ }^{a}$ Federal University of São Paulo, Department of Bioscience, Avenue Ana Costa 95, Santos 11050-240, Brazil \\ ${ }^{b}$ Federal University of São Carlos, Department of Genetics and Evolution, Rod Washington Luis Km 235, São Carlos 13565-905, Brazil \\ 'University of São Paulo, School of Pharmaceutical Sciences of Ribeirão Preto, Department of Clinical Analysis, Toxicological and Bromatological, \\ Avenue do Café 95, Ribeirão Preto, Brazil 14049-900, Brazil \\ dState University of Campinas, Department of Genetics and Evolution, Cidade Universitária Zeferino Vaz, Campinas 13083-970, Brazil \\ eBrazilian National Center for Research in Energy and Materials, Brazilian Biosciences National Laboratory, Giuseppe Máximo Scolfaro 10.000, \\ Campinas 13083-970, Brazil \\ ${ }^{\mathrm{f}}$ Federal University of São Carlos, Department of Physiotherapy, Rod Washington Luis Km 235, São Carlos 13565-905, Brazil
}

\begin{abstract}
This study investigates the histological modifications produced by low level laser therapy (LLLT) on the first day of bone repair, as well as evaluates the LLLT effects on collagen expression on the site of a fracture. Twenty Wistar rats were distributed into a control group (CG) and a laser group (LG). Laser irradiation of Ga-AlAs laser $830 \mathrm{~nm}, 30 \mathrm{~mW}, 94 \mathrm{~s}, 2.8 \mathrm{~J}$ was performed in five sessions. Animals were euthanized on day 5 postsurgery. Histopathological analysis showed that LLLT was able to increase deposition of granulation tissue and newly formed bone at the site of the injury. In addition, picrosirius analysis showed that collagen fiber organization in the LG was enhanced compared to CG. Microarray analysis demonstrated that LLLT produced an upregulation type I collagen (COL-I). Immunohistochemical analysis revealed that the subjects that were treated presented a higher immunoexpression of COL-I. Our findings indicated that LLLT improves bone healing by producing a significant increase in the expression of collagen genes. ๑ 2016 Society of Photo-Optical Instrumentation Engineers (SPIE) [DOI: 10.1117/1.JBO.21.8.088001]
\end{abstract}

Keywords: bone regeneration; low level laser therapy; microarray; collagen.

Paper 160147RR received Mar. 8, 2016; accepted for publication Aug. 2, 2016; published online Aug. 19, 2016.

\section{Introduction}

Bone is a mineralized connective tissue and it constitutes cells (osteocytes, osteoblasts, and osteoclasts), inorganic molecules (calcium, phosphate, and hydroxyapatite), and organic matrix components (collagen and noncollagenous matrix proteins). ${ }^{1}$ Collagen is the main component of the organic phase of the bone tissue. ${ }^{2}$ It represents more than $90 \%$ of the organic matrix and it plays a central role in bone architecture, supporting bone mineralization. ${ }^{3-5}$ Additionally, type I collagen has an important function during the bone repair process, and it is specially deposited in granulation tissues by fibroblastic and osteoblastic cells. ${ }^{6,7}$ There is evidence that the presence of collagen during the healing process is responsible for organization of primary trabecular bone and mature bone. ${ }^{8,9}$ Likewise, collagen may be an influential contributor to the strength and stiffness of the whole bone or bone matrix. ${ }^{10}$ However, during this complex process, collagen production can be affected by mutations in the synthesis of type I collagen genes (COL-I) ${ }^{11}$ and such mutations could be related to the decrease of collagen deposition, which may severely compromise the bone mineralization and its mechanical capacity to sustain postyield deformation and, therefore, absorb energy prior to fracture. ${ }^{12}$ In addition, there have

*Address all correspondence to: Carla Roberta Tim, E-mail: carlinha_tim @ hotmail.com been reports showing a direct link between a polymorphism in the COL-I gene and increased risk of bone fracture. ${ }^{13}$

In view of the aforementioned, collagen synthesis has a relevant role during the bone repair and, therefore, therapies able to induce the deposition of collagenous matrix could be beneficial to treat nonunion fractures. ${ }^{14}$ One promising treatment is the use of low level laser therapy (LLLT) due to its stimulatory effect on tissue metabolism by stimulating cell proliferation, ${ }^{15}$ stimulating collagen production by fibroblasts and osteoblasts, ${ }^{16}$ and accelerating the bone repair. ${ }^{17} \mathrm{~A}$ recent study demonstrated that LLLT increased the osteoblastic proliferation and gene expression of COL-I as well as other osteoblastic markers in osteoblast cells derived from the midpalatal suture. ${ }^{18}$ Moreover, Bossini et al. ${ }^{19}$ showed that LLLT was able to increase the collagen deposition in bone repair in osteoporotic rats.

Despite the encouraging data concerning the potential effects of LLLT to induce newly formed bone, the molecular and cellular mechanisms by which this therapy acts on collagen deposition in the initial periods of repair are not fully understood, since most studies have evaluated its effects in in vitro study and in vivo study on intermediate and late periods of bone healing. ${ }^{8,19}$ Moreover, there is a lack in the literature of studies using sophisticated techniques in gene expression analysis (i.e., microarrays), which is possible to examine the global gene

$1083-3668 / 2016 / \$ 25.00$ @ 2016 SPIE 
expression and to investigate entire pathways that command biological processes in the initial periods. Thus, the present study can provide significant insights of the bone healing process. Therefore, it was hypothesized that the treatment of bone defects with LLLT on early stages of fracture healing, which is the most active period in the recruitment of cells, could stimulate proliferation of fibroblasts and osteoblasts; consequently, collagen deposition, which can contribute to bone mineralization, may provide a treatment with additional advantages for clinical use. Still, this therapy could benefit the treatment of larger bone defects and fractures with inadequate or interrupted vascularization, a delay in the bone healing process or even a nonunion may happen. Thus, the present study aims to evaluate the effects of LLLT on collagen expression on the site of bone defect, 5 days postsurgery. For this purpose, cDNA microarray analysis was utilized to identify regulated gene expression after LLLT and the molecular mechanisms involved in the effects. Also, histological analysis was used to evaluate the area of newly formed bone in an experimental tibial bone defect model in rats.

\section{Materials and Methods}

The animal handling and surgical procedures were strictly conducted according to the Guiding Principles for the Use of Laboratory Animals. All procedures were approved by Institutional Animal Ethics Committee of the Federal University of São Carlos (Protocol 010/2011).

\subsection{Surgical Procedure}

Twenty male rats (Rattus norvegicus, Wistar) (350 $\pm 10 \mathrm{~g} ; 12$ weeks) were used in this study. After intraperitoneal anesthesia with ketamine/xylazine $(80 / 10 \mathrm{mg} / \mathrm{Kg})$, a $1-\mathrm{cm}$ incision was made in the skin to expose the tibia and after that, a standardized 3.0-mm-diameter bone defect was created bilaterally by using a motorized round drill (BELTEC ${ }^{\circledR}$, Araraquara-SP, Brazil), under constant physiologic saline solution irrigation. Thereafter, the cutaneous flap was joined and sutured with resorbable Vicryl ${ }^{\circledR}$ 5-0 (Johnson \& Johnson, St. Stevens-Woluwe, Belgium). In order to minimize postoperative discomfort, the animals received analgesia (i.m., $0.05 \mathrm{mg} / \mathrm{kg}$ buprenorphine Temgesic; Reckitt Benckiser Health Care Limited, Schering Plough, Hoddesdon, UK) and were returned to their cages. The health status of the rats was monitored daily.

\subsection{Experimental Groups}

Animals were randomly divided into two groups of 10 animals each:

1. Control group (CG): bone defects without any treatment.

2. Laser group (LG): bone defects treated with laser $830 \mathrm{~nm}$.

\subsection{Low Level Laser Therapy}

A laser (Thera laser, DMC ${ }^{\circledR}$ São Carlos, Brazil) of $0.6 \mathrm{~mm}$ beam diameter with a continuous wavelength $(\lambda)$ of $830 \mathrm{~nm}$, $0.028 \mathrm{~cm}^{2}$ spot area, $30 \mathrm{~mW}, 94 \mathrm{~s}, 2.8 \mathrm{~J}, 1.071 \mathrm{~W} / \mathrm{cm}^{2}$, and $100 \mathrm{~J} / \mathrm{cm}^{2}$ was used. Laser irradiation started immediately after the surgery at one transcutaneous point, above the site of the injury, and it was performed with an interval of $24 \mathrm{~h}$
Table 1 LLLT parameters.

\begin{tabular}{lc}
\hline Laser & Ga-Al-As \\
\hline Equipment & Thera laser, $\mathrm{DMC}^{\circledR}$ São Carlos, Brazil \\
Frequency & Continuous \\
Power & $30 \mathrm{~mW}$ \\
Power density & $1.071 \mathrm{~W} / \mathrm{cm}^{2}$ \\
Spot size (cm ${ }^{2}$ ) & $0.028 \mathrm{~cm}^{2}$ \\
Energy density & $100 \mathrm{~J} / \mathrm{cm}^{2}$ \\
Time per point & $94 \mathrm{~s}$ \\
Energy & $2.8 \mathrm{~J}$ \\
Number of points & 1 \\
Method & Transcutaneous \\
\hline
\end{tabular}

between each session, totaling five sessions (Table 1). Laser irradiation was performed, at one point per tibia, above the site of the injury (using the punctual contact technique) and was applied bilaterally.

\subsection{Retrieval of Specimens}

Five days postsurgery ( $24 \mathrm{~h}$ after the last laser application), all animals were euthanized individually by carbon dioxide asphyxia. Then, both tibias were removed and rapidly dissected. The left tibias were immediately fixed in $10 \%$ formaldehyde (Merck, Darmstadt, Germany) for $24 \mathrm{~h}$. The right tibias were frozen in liquid nitrogen and stored in freezer at $-80^{\circ} \mathrm{C}$ until microarray analysis was carried out.

\subsection{Histological Analysis}

After fixed, the left tibias were decalcified in 4\% diamine tetraacetic acid (Merck, Darmstadt, Germany) for 40 days. Then, the sample was dehydrated in a graded series of ethanol and embedded in paraffin. Therefore, thin sections $(5 \mu \mathrm{m})$ were prepared in the longitudinal plane, using a micrometer (Leica RM -2145, Germany).

\subsection{Qualitative Analysis}

For the qualitative histopathological analysis, the laminas were stained with hematoxylin and eosin (H.E stain, Merck, Darmstadt, Germany). Histopathological evaluation was performed in a blinded manner, under a light microscope (Olympus, Optical Co. Ltd, Tokyo, Japan). Any changes in the bone defect, such as presence of blood clots, fibrin, inflammatory processes, granulation tissue, woven bone, or even tissues undergoing hyperplastic, metaplastic, and/or dysplastic transformation were investigated in each animal. In addition, histological sections stained by the picrosirius-polarization method were viewed under polarized light to assess the structural changes in the neoforming trabecular matrix. This method allows a qualitative and quantitative evaluation of the stage of bone matrix organization based on the birefringence of the collagen fiber bundles after staining with picrosirius-red and hematoxylin. 
To perform the analysis, the software ImageJ (version 1.36) was used, and it is possible to identify the brightness of birefringence by calculating the intensity in "pixels" of color given picrosiriusred under polarized light. The intensity of pixels is proportional to the organization of collagen fibers. The collagen fibers are thicker and strongly birefringent presents stained in shades of orange to red, which is the most anisotropic collagen. ${ }^{8}$ Ten fields were evaluated in the defect region, extension of $100 \times$, allowing the analysis of the entire focus of the lesion. The values corresponding to each field were added, resulting in the birefringence of collagen fibers of the bone defect per animal.

\subsection{Morphometry Analysis}

Sections stained with H.E were used to perform the morphometry of the area of newly formed bone. The analysis was measured in a blind fashion by two observers, using the image analysis system Motican 5.0. Five areas of bone defect region were selected and named $\mathrm{C} 1$ (upper region of the border), $\mathrm{C} 2$ (right border), C3 (bottom region of the border), C4 (left border), and C5 (central region of the bone defect). The amount of newly formed bone was determined in $\mu \mathrm{m}^{2}$ in each region, and the total newly formed bone was represented as $\mathrm{C} 1+\mathrm{C} 2+\mathrm{C} 3+\mathrm{C} 4+\mathrm{C} 5$.

\subsection{Immunohistochemistry}

After deparaffinization and rehydration in graded ethanol, each specimen was pretreated in a Steamer with buffer Diva Decloaker (Biocare Medical, California) for 5 min for antigen retrieval. The material was preincubated with $0.3 \%$ hydrogen peroxide in phosphate-buffered saline (PBS) solution for $30 \mathrm{~min}$ in order to inactivate endogenous peroxidase and then blocked with 5\% normal goat serum in PBS solution for $20 \mathrm{~min}$. Three sections of each specimen were incubated with anticollagen (COL-I, Cat. no. sc-59772) polyclonal primary antibody at a concentration of 1:1200 (Santa Cruz Biotechnology, Santa Cruz, California). Incubation was performed overnight at $4^{\circ} \mathrm{C}$ in refrigerated environment. Afterward, two washes were done in PBS for $10 \mathrm{~min}$. Then, incubation of the sections was performed making use of biotin-conjugated secondary antibody anti-rabbit immunoglobulin $\mathrm{G}$ (IgG) (Vector Laboratories, Burlingame, California) at a concentration of 1:200 in PBS for $30 \mathrm{~min}$. The sections were washed twice with PBS followed by the application of preformed avidin/biotin complex conjugated to peroxidase (Vector Laboratories, Burlingame, California) for $30 \mathrm{~min}$. The bound complexes were visible by the application of a $0.05 \%$ solution of $3-3^{\prime}$-diaminobenzidine solution and counterstained with Harris hematoxylin. In order to carry out control studies of the antibodies, the serial sections were treated with rabbit IgG (Vector Laboratories, Burlingame, California) at a concentration of 1:200 instead of the primary antibody. Furthermore, internal positive controls were performed with each staining bath.

Collagen immunoexpression was assessed both qualitatively (presence of the immunomarkers) and semiquantitatively in five predetermined fields using a light microscopy (Leica Microsystems AG, Wetzlar, Germany) according to a previously described scoring scale ranging from 1 to $4(1=$ absent, $2=$ slight, $3=$ moderate, and $4=$ intense) for immunohistochemical analysis. ${ }^{20}$ The analysis was performed by two observers (PB and $\mathrm{CT}$ ), in a blinded manner.

\subsection{RNA Sample Preparation}

Total RNA was isolated from the right tibias using the TRIzol ${ }^{\circledR}$ reagent (Invitrogen, Carlsbad, California) according to the manufacturer's instructions. After the RNA isolation, the samples were purified using the illustra RNAspin Mini RNA Kit (GE Healthcare Life Sciences) according to the manufacturer's instructions, quantified by NanoVue spectrophotometer (GE Healthcare Life Sciences). The quality and integrity of the total RNA were confirmed with an Agilent 2100 Bioanalyzer (GE Healthcare Life Sciences) and samples presenting RNA integrity numbered $\geq 8$ were used for cRNA synthesis.

\subsection{Microarray Hybridizations}

Agilent whole rat genome microarray $4 \times 44 \mathrm{~K}$ was used to perform microarray hybridizations. The labeling and microarray hybridizations were performed by Agilent using a twocolor microarray-based gene expression analysis (Agilent Technologies). Briefly, for cDNA synthesis and labeling, $200 \mathrm{ng}$ of total RNA was used. After, cDNA was transcribed into cRNA, and it was labeled using Agilent low RNA input fluorescent linear amplification kit (Agilent Technologies, Santa Clara, California). Then, cRNA labels were purified, mixed with hybridization buffer, and hybridized to an Agilent whole rat genome microarray $4 \times 44 \mathrm{~K}$ for $17 \mathrm{~h}$ at $65^{\circ} \mathrm{C}$, according to the manufacturer's instructions. The hybridized microarrays were washed as the manufacturer's washing protocol (Agilent Technologies, Santa Clara, California). After hybridization, microarrays were sequentially washed: $1 \mathrm{~min}$ at room temperature in GE wash buffer 1 (Agilent Technologies), then $1 \mathrm{~min}$ at $37^{\circ} \mathrm{C}$ in GE wash buffer 2 (Agilent Technologies), followed by $10 \mathrm{~s}$ in acetonitrile wash (Agilent Technologies), and finally $30 \mathrm{~s}$ in stabilization and drying solution wash (Agilent Technologies). Afterward, microarray slides were scanned using GenePix ${ }^{\circledR}$ 4000B microarray scanner (Molecular Devices) while simultaneously scanning the $\mathrm{Cy} 3$ and Cy5 channels at a resolution of $5 \mu \mathrm{m}$. Laser was set at $100 \%$ and PMT gain was automatically adjusted for each slide using the program GenePix 4000B according to the intensity of the signal in each array.

\subsection{Microarray Data Analysis}

Microarray data analysis was performed as described by Castro et al. ${ }^{21}$ Data files were generated using Agilent's feature extraction software (version 11.5, Agilent) and the default parameters, which include Lowess-based signal normalization. The dye-normalyzed values generated in the feature extraction data files were used to upload the software express converter (version 2.1, TM4 available at Ref. 22), which conveniently converts the Agilent file format to $\mathrm{MeV}$ (multiexperiment view) file format compatible to the TM4 softwares for microarray analysis (available at Ref. 23). The MeV files were then uploaded in the MIDAS software where the resulting data were averaged from replicated genes on each array, from three biological replicates, taking a total of three intensity data points for each gene. The $\mathrm{MeV}$ files generated were then loaded in $\mathrm{MeV}$ software where differentially expressed genes were identified using one-class $t$-test $(p<0.01)$. Significantly different genes were those whose mean $\log 2$ expression ratio over all included samples was statistically different from 0 , which indicates the absence of gene modulation. 


\subsection{Functional Analyses Using Ingenuity Pathways Analysis Software}

A network analysis was performed using the ingenuity pathways analysis (IPA) (Ingenuity Systems) ${ }^{24}$ algorithm. The lists of differentially expressed genes were entered into the IPA software to explore relevant biological networks and to assess interactions with other genes. A hypothetical global gene interaction network was constructed, showing the most relevant direct and indirect connections of genes found to be regulated under LLLT.

\subsection{Quantitative Real-Time Polymerase Chain Reaction}

Real-time polymerase chain reaction (RT-PCR) was performed to confirm the differential expression results obtained by the microarray experiments. Total RNA was extracted and purified using the experimental protocols described above. One microgram of total RNA was reverse-transcribed into cDNA and followed by RT-PCR amplification using rats gene-specific primers (Table 2), which were designed by primerexpress software (Applied Biosystems, Foster City, California). The optimized PCR conditions were: initial denaturation at $94^{\circ} \mathrm{C}$ for $10 \mathrm{~min}$, followed by 40 cycles consisting of denaturation at $94^{\circ} \mathrm{C}$ for $15 \mathrm{~s}$, annealing at $60^{\circ} \mathrm{C}$ for $1 \mathrm{~min}$, and extension at $72^{\circ} \mathrm{C}$ for $45 \mathrm{~s}$, with a final extension step at $72^{\circ} \mathrm{C}$ for $2 \mathrm{~min}$. Negativecontrol reactions with no template (deionized water) were also included in each run. All samples were amplified simultaneously in duplicate in one assay run. Analysis of relative gene expression was performed using the 2- $\Delta \Delta \mathrm{CT}$ method. RPS18 was used as a housekeeping gene to normalize our expression data.

\subsection{Statistical Analysis}

The normality of all variables distribution was verified using Shapiro-Wilk's W test. For morphometric and immunohistochemical analysis, comparisons among groups were performed using $t$-test. STATISTICA version 7.0 (data analysis software system-StatSoft Inc.) was used to carry out the statistical analysis. Values of $p<0.05$ were considered statistically significant.

\section{Results}

\subsection{Histological Analysis}

An overview of the representative histological sections of all experimental groups is shown in the Fig. 1. For CG, 5 days after the surgery, the bone defect area was filled by inflammatory infiltrate, granulation tissue, and immature newly formed bone [Fig. 1(a)]. At the same experimental period, for the LG, inflammatory infiltrate was presented only in the central region of the defect. The rest of the defect was filled by some areas of granulation tissue and bone ingrowth [Fig. 1(b)]. In addition, picrosirius analysis showed that collagen fiber organization in the LG was enhanced compared to the CG, demonstrated by the increased thickness of the colored areas [Figs. 2(a) and 2(b)].

\subsection{Morphometric Analysis}

Figure 3 shows that the animals exposed to laser therapy presented a statistically significant increase in the newly formed bone at the defect area compared to the CG (Fig. 2).

\subsection{Immunohistochemical Analysis}

Qualitative immunohistochemical analysis demonstrated that COL-I expression was detected in granulation tissue, osteoblast, and bone matrix for both groups [Fig. 4(a)]. However, for the LG, the immunoreactivity of COL-I was mainly observed in osteoblast and newly formed bone, when compared to the CG. Semiquantitative analysis revealed a significant increase in COL-I immunoexpression for the LG compared to the CG, 5 days after surgery [Fig. 4(b)].

\subsection{Microarray Analysis}

In order to examine genes modulated by LLLT during bone repair on a genome-wide basis, microarray expression profiling was carried out on skeletal tissues. Thus, a total of 382 genes modulated were identified after laser irradiation. It was found that 127 genes downregulate and 255 genes upregulate.

\subsection{Functional Network Analysis}

In order to analyze these expression data, a reliable new bioinformatics approach, IPA (Ingenuity, California) was applied to set up a potential network, which is based on the regulated genes in order to identify the molecular events and further unveil the molecular mechanisms regulating the processes of LLLT effects in bone. In order to start building networks, IPA queries the ingenuity pathways knowledge base for interactions between the regulated genes and the genes stored in the database. The networks were identified and ranked according to the score $p$-score $=-\log 10$ ( $p$-value $)$. The score takes into account

Table 2 Real-time PCR primers.

\begin{tabular}{lrr} 
Gene & Forward primer & Reverse primer \\
\hline RPS18 & GTGATCCCCGAGAAGTTTCA & AATGGCAGTGATAGCGAAGG \\
PTGER2 & GAACTGCGAGAGTCGTCAGTATCTC & CCCCGGCCGTGAACAT \\
IL1R1 & AAGTGGAATGGGTCGGAAATT & TGAAGGGTGTTCCAAAAACTGA \\
ANGPT4 & GGCATCTACTATCCGGTTCATCA & CATGCGTGTGCCATGCA \\
PDGFD & TATGCTCATTGGATGCCTTGTC & TGCTGCTATCGGGACACTTTT \\
FGF2 & AAGGATCCCAAGCGGCTCTA & CGGCCGTCTGGATGGA \\
\hline
\end{tabular}




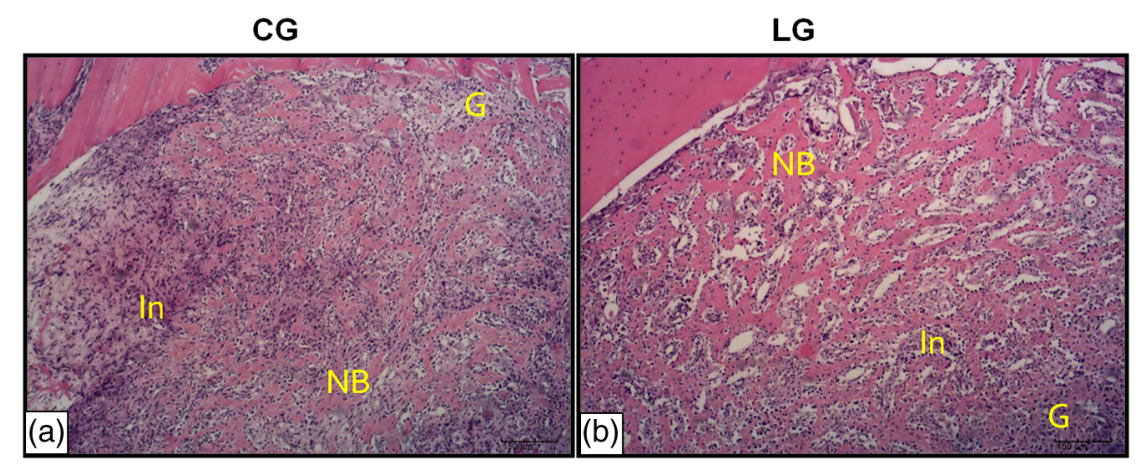

Fig. 1 Representative sections of hematoxylin and eosin stain. Newly formed bone (Nb), granulation tissue (G), inflammatory infiltrate (In). (a) $C G$ = control group, (b) $L G=$ laser group. Scale bar $=150 \mu \mathrm{m}$.

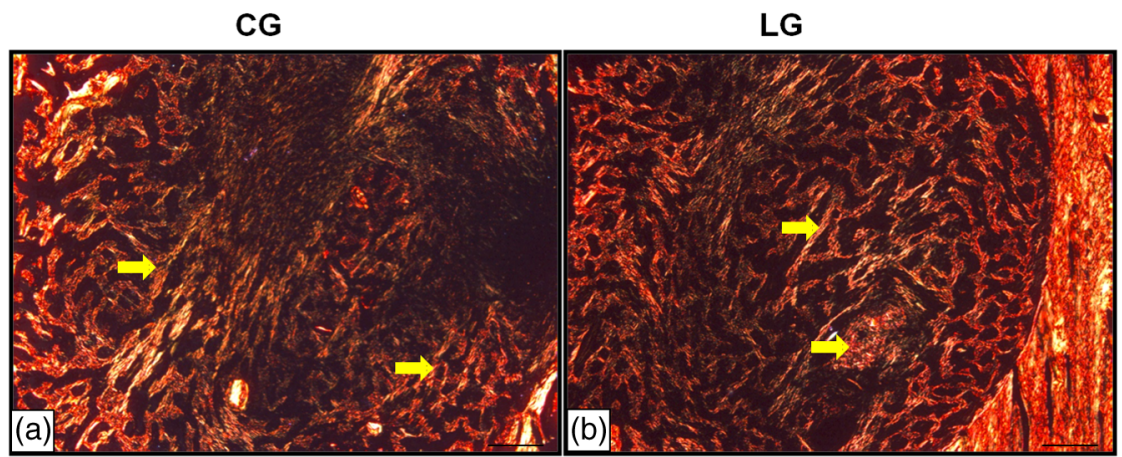

Fig. 2 Representative sections of picrosirius red stain. Photomicrographs using polarized light illustrating the collagen. Arrow indicates the yellowish and reddish birefringent zones. (a) CG = control group, (b) LG $=$ laser group. Scale bar $=150 \mu \mathrm{m}$.

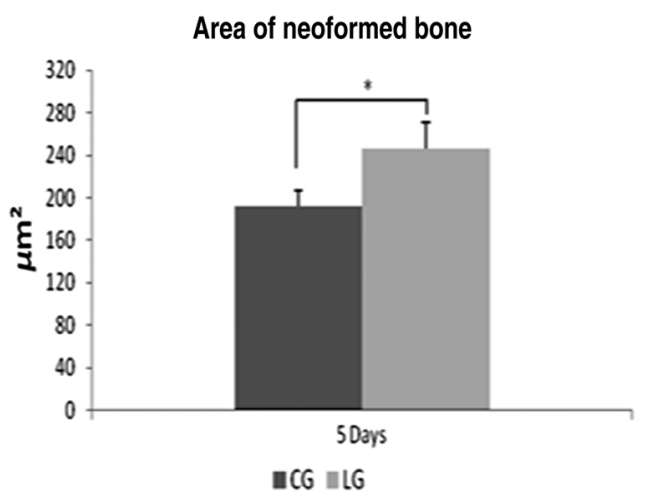

Fig. 3 Means and SD of the newly formed bone tissue of bone area $\left(\mu \mathrm{m}^{2}\right)$ of the defect after treatments. Significant differences of $p<0.001$ are represented by a single asterisk $\left({ }^{*}\right)$.

the number of network eligible molecules in the network and its size, as well as the total number of network eligible molecules analyzed and the total number of molecules in the ingenuity knowledge base, which could potentially be included in networks. A score $>3(p<0.001)$ indicates a $>99.9 \%$ confidence that a gene network was not generated by chance alone. Furthermore, the IPA score can be interpreted as the probability of getting a network containing at least the same number of network eligible molecules by chance when randomly picking genes that can be in networks from the ingenuity knowledge base. Thus, the major biological processes identified for increased gene expressions related to inflammatory responses, connective tissue development, and skeletal and muscular system activity were investigated (Table 3 ).

In view of the aforementioned, several functional groupings of differentially expressed genes were identified. The up- and downregulated connective tissue development and function genes were further examined and revealed some COX-2-related genes, interleukins, growth factors, angiogenic, and osteogenic signaling and provided an initial analysis. However, this report focused on expression of bone collagen of early events of bone healing and the downregulation and upregulation connective tissue genes were examined and revealed the significantly upregulated of type I collagen gene. The altered expression of this gene suggests that LLLT acts on the stimulation of fibriblasts and osteoblast, consequently in bone forming.

\subsection{Validation of Microarray Data by Real-Time Polymerase Chain Reaction}

Quantitative real-time PCR (qPCR) is a commonly used validation tool for confirming gene expression results obtained from microarray analysis. Thus, qPCR was performed to validate our results of the microarray assays. The genes were selected according to the canonical pathway analysis and the expression profiles of the genes confirmed the microarray results. Our PCR results were consistent with the pathway prediction, showing that the relative mRNA levels of those osteogenic genes were modulated by LLLT (Fig. 5). 


\section{COL-I immunoexpression}

(a) Qualitative immunohistochemical

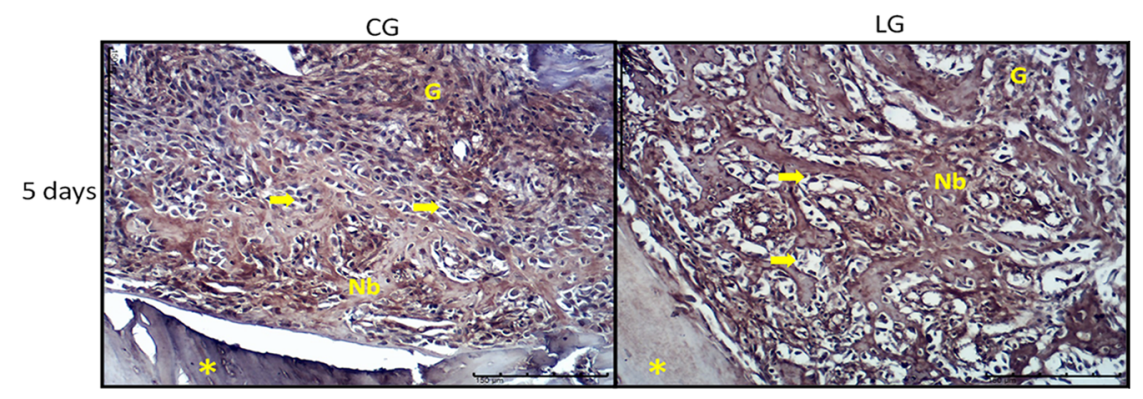

(b) Semi quantitative immunohistochemical

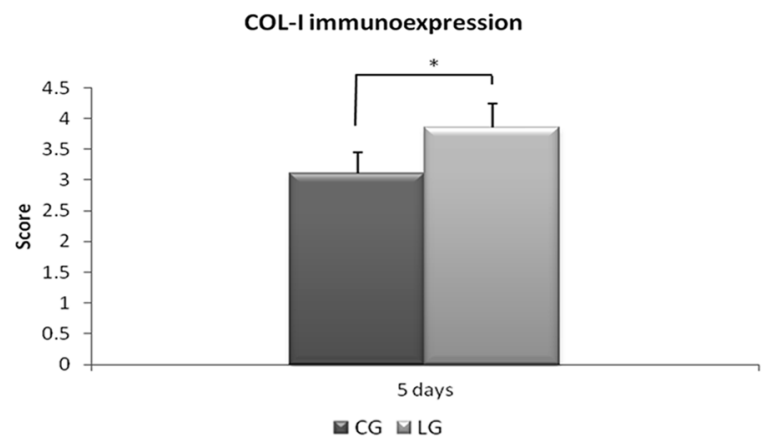

Fig. 4 (a) representative sections of COL-I immunohistochemistry. Newly formed bone (Nb), granulation tissue $(\mathrm{G})$, osteoblast, and $(\mathrm{Ob})$ intact bone $\left(^{*}\right)$. (b) means and standard error of the mean of scores immunohistochemistry of COL-I. Significant differences of $p<0.05$ are represented by a single asterisk $\left(^{*}\right)$.

Table 3 Top genetic network.

Network functions

Score

Day 5

Connective tissue development and function

Skeletal and muscular system development and function, cell-to-cell signaling and interaction Cell-to-cell signaling and interaction, inflammatory response, and gene expression

Cell cycle, DNA replication, recombination, and repair, 15 cellular assembly, and organization

Cell morphology, cellular assembly and organization, 12 and cellular development

\section{Discussion}

This study aimed to investigate the histological modifications produced by LLLT and to study the collagen deposition in the initial stages of bone healing. The main findings showed that LLLT increased the deposition of granulation tissue and newly formed bone. In addition, the COL-I immunoexpression was also increased by LLLT. Furthermore, our results showed that LLLT produced a significant increase in the expression of type I collagen genes.

Several studies have shown that LLLT is able to induce cell proliferation, accelerate tissue metabolism, and produce collagen synthesis on the process of tissue repair. ${ }^{20,25-27}$ This study suggest that LLLT was able to attract the osteoprogenitor cells and induce their differentiation into fibroblasts and osteoblasts, thus promoting collagen deposition in early phases of the bone repair process. Collagen deposition was investigated by picrosirius analysis, which allowed the correlation between the growth of primary (trabecular) bone area with the collagen fibers deposition. This coloration is used to investigate the reddish and yellowish birefringence, which is indicative of type I and III collagen. These results corroborate with others studies that used picrosirius analysis and demonstrated that LLLT stimulated the growth of the trabecular area and hastened the organization of matrix collagen during the first week of bone repair. ${ }^{8}$ At the same way, Bossini et al. ${ }^{19}$ demonstrated that irradiated animals presented a higher deposition of collagen fibers and a better organization of these fibers when compared to the CG.

In parallel with these changes, the morphometric analysis showed that the LG was capable of increasing the deposition of newly formed bone, when compared to the CG. These findings are in line with the results of Fernandes et al., ${ }^{28}$ which showed that LLLT induced a recruitment of inflammatory cells and increased newly formed bone in the initial phases of bone consolidation. Additionally, effects of LLLT on the collagen synthesis were also demonstrated by the increased immunoexpression of COL-I in the LG when compared with the CG. Also, recent studies demonstrated that LLLT produced an increase of gene expression of COL-I. ${ }^{17,18}$ Likewise, several studies showed that LLLT increased COL-I immunoexpression during the early phases of tissue repair. ${ }^{29,30}$

Collagens constitute a family of proteins, with more than 27 forms, and in the bone tissue the types I, II, III, V, VI, and XI collagen are present. ${ }^{10,31,32}$ However, it is well known that the 

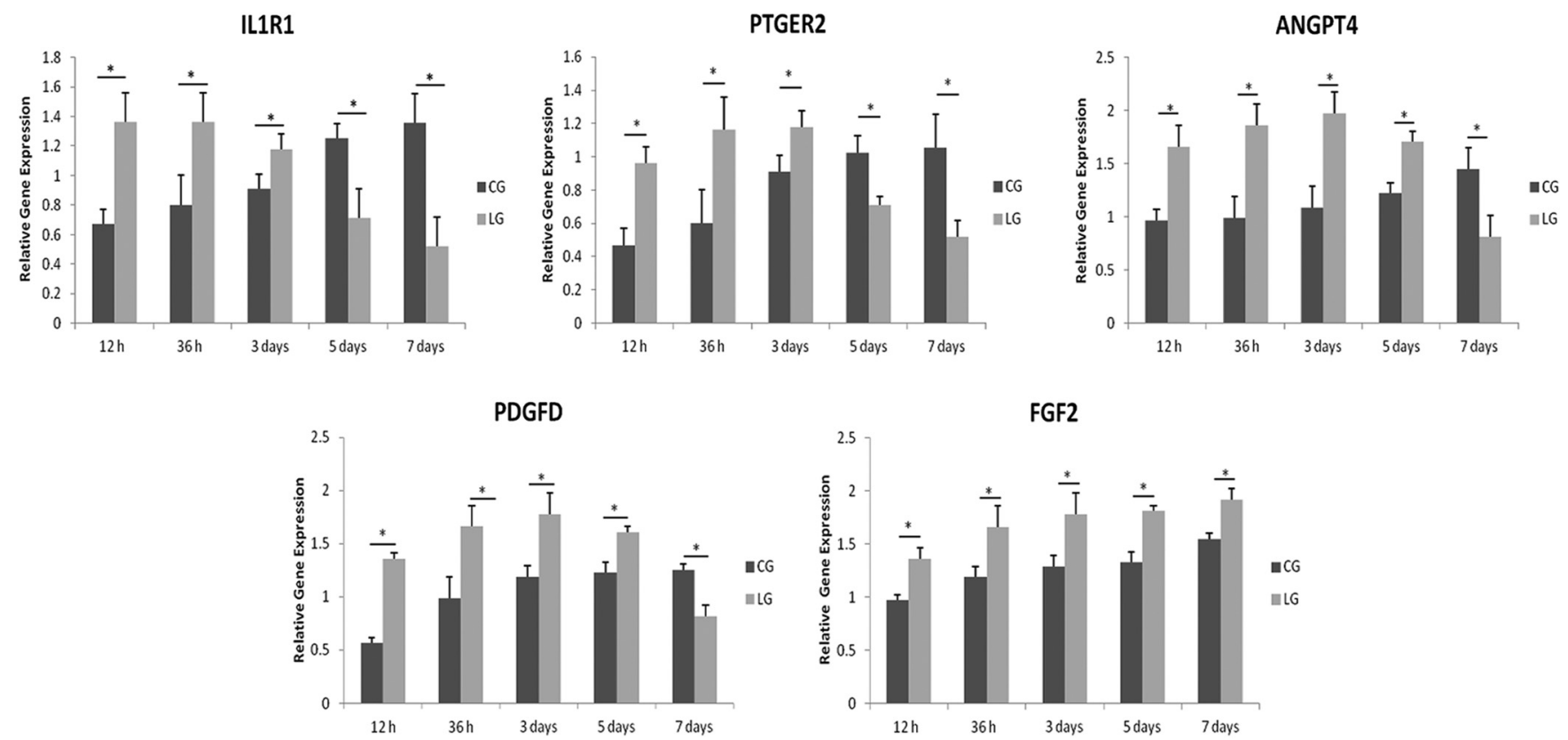

Fig. 5 Validation of up- and downregulated gene expression by real-time RT-PCR in irradiated animals. The results are normalized as a ratio of each specific mRNA signal to the RPS18 gene signal within the same sample and the values expressed. The significance of differences was determined using Student's t-test. ${ }^{*} p<0.05$.

type I collagen has the most abundant and important collagen in the bone tissue. ${ }^{10}$ In order to further investigate the action of the LLLT on bone repair, members of the collagen family expressed locally during the bone repair process were evaluated. In the present study, microarray analysis showed that LLLT produced an upregulation of type I collagen gene. This result suggests that the increase in gene expression of collagen, in special type I, could have contributed to the healing process and accelerated the bone repair process, once COL-I is the major structural protein and the main extracellular matrix protein for calcification. ${ }^{10,33}$ Likewise, previous studies demonstrated that LLLT $(830 \mathrm{~nm})$ increased the osteoblastic proliferation and increased the expression of osteoblastic markers, such as gene expression of COL-I, runt-related transcription factor 2, osteocalcin, and alkaline phosphatase.$^{18}$ Moreover, other studies showed that LLLT (904 and $830 \mathrm{~nm}$ ) could induce enhanced repair by increasing the expression of procollagen type I and III mRNA ${ }^{34,35}$ and increasing collagen synthesis. ${ }^{19}$

It is known that the bone repair process constitutes a dynamic tissue reaction, which comprises different phenomena, such as inflammation, cell proliferation, and synthesis of elements of the extracellular matrix, including collagen. Synthesis of collagen fibers plays a central role in bone architecture, once the bone mineral crystals are deposited among collagen fibrils, which contribute to the bone mineralization. ${ }^{3,5,36}$ Mutations in the amino acid sequence of collagen, as in osteogenesis imperfecta, can seriously impair the formation, orientation, and organization of the apatite crystals, which significantly increases the brittleness of bone. ${ }^{37}$ Therefore, it is clear that there is a relationship between the fibril structure and mineral formation. ${ }^{36}$

According to several authors, ${ }^{19,35,38,39}$ some main biostimulation effects of LLLT in the process of tissue repair include induction of mitotic activity of epithelial cells and fibroblasts, and the stimulation of collagen production by those cells.
There are several mechanisms through which LLLT could induce mitotic activity of fibroblasts. LLLT stimulates the production of basic fibroblast growth factor (bFGF), which is a multifunctional polypeptide secreted by fibroblasts. It is not only able to induce fibroblast proliferation but also differentiation, and it affects the immune cells that secrete cytokines and other regulatory growth factors of fibroblasts. In addition, it has been reported that LLLT increases the levels of ascorbic acid in fibroblasts, thus increasing the formation of hydroxyproline and, consequently, the production of collagen, since ascorbic acid is a cofactor required for hydroxylation of proline during collagen synthesis. $^{40}$

In addition, it is possible to suggest that these results could be related to the laser parameters used in the present study. There are reports in the literature proposing that the wavelength of $830 \mathrm{~nm}$, which was used in this study, could be advantageous to induce the increase fibroblast cell proliferation that could increase the collagen synthesis. ${ }^{41,42}$ Similarly, it is possible to suggest that the laser energy $(2.8 \mathrm{~J})$ used in this study had a positive result on bone metabolism at the site of the injury. Our results corroborate those of Fernandes et al. and Bossini et al. that used 830-nm laser, at the energy of 2.8 and $3.4 \mathrm{~J}$, respectively, to investigate the effects of LLLT during the process of bone healing and showed that LLLT was able to induce osteogenic genes and collagen deposition. Our results demonstrated that LLLT could increase the newly formed bone. These findings could be related with COL-I expression once the literature demonstrated that production of type I collagen is one of the earliest events associated with osteoblastic differentiation. ${ }^{43}$ Thus, it could propose that the upregulation of this gene may indicate the presence of more active fibroblasts and osteoblasts, which can be related to the increase of granulation tissue and newly formed bone observed in the histological analysis in the LLLT group. 
Taken together, the results of the present study showed that LLLT might be a promising therapy to improve bone consolidation in the initial period of repair by modulating the expression of COL-I, which may contribute to stimulate bone cells and increase newly formed bone. As this study was limited to a short-term evaluation, information on the influence of LLLT on gene expression in long-term analysis still needs to be provided.

\section{Conclusion}

The results found in the present study indicate that LLLT was efficient in modulating the inflammatory process, stimulating bone metabolism, and accelerating new bone formation and collagen deposition at the site of the injury. In addition, LLLT produced a significant increase in the expression of COL-I expression, which contributes to the bone mineralization. This fact may explain mechanisms that LLLT acts on bone healing. Therefore, these data highlight the potential of LLLT to be used as a therapeutic approach for bone regeneration.

\section{Acknowledgments}

We would like to acknowledge the contributions of Brazilian funding agency Fapesp (2010/15335-0) and the Center for Computational Engineering and Sciences at UNICAMP SP Brazil (FAPESP/CEPID and project \#2013/08293-7) for the financial support of this research. None of the authors have financial conflicts of interest to disclose.

\section{References}

1. B. Buehring et al., "Glucocorticoid-induced osteoporosis: an update on effects and management," J. Allergy Clin. Immunol. 132(5), 1019-1030 (2013).

2. A. Alford, K. M. Kozloff, and K. D. Hankenson, "Extracellular matrix networks in bone remodeling," Int. J. Biochem. Cell Biol. 65, 20-31 (2015).

3. E. Mackie, "Osteoblasts: novel roles in orchestration of skeletal architecture," Int. J. Biochem. Cell Biol. 35(9), 1301-1305 (2003).

4. P. Proff and P. Römer, "The molecular mechanism behind bone remodelling: a review," Clin. Oral Invest. 13(4), 355-362 (2009).

5. L. J. Raggatt and N. C. Partridge, "Cellular and molecular mechanisms of bone remodeling," J. Biolog. Chem. 285(33), 25103-25108 (2010).

6. D. M. Lawton et al., "Mature osteoblasts in human non-union fractures express collagen type III," Mol. Pathol. 50(4), 194-197 (1997).

7. S. W. Volk et al., "Type III collagen regulates osteoblastogenesis and the quantity of trabecular bone," Calcified Tissue Int. 94(6), 621-631 (2014).

8. I. Garavello-Freitas et al., "Low-power laser irradiation improves histomorphometrical parameters and bone matrix organization during tibia wound healing in rats," J. Photochem. Photobiol. B 70(2), 81-89 (2003).

9. T. E. Kruger, A. H. Miller, and J. Wang, "Collagen scaffolds in bone sialoprotein-mediated bone regeneration," Sci. World J. 20131-6 (2013).

10. S. Viguet-Carrin, P. Garnero, and P. D. Delmas, "The role of collagen in bone strength," Osteoporosis Int. 17(3), 319-336 (2006).

11. J. Myllyharju and K. I. Kivirikko, "Collagens and collagen-related diseases," Ann. Med. 33(1), 7-21 (2001).

12. D. Vashishth, "The role of the collagen matrix in skeletal fragility," Curr. Osteoporosis Rep. 5(2), 62-66 (2007).

13. D. B. Burr, "The contribution of the organic matrix to bone's material properties," Bone 31(1), 8-11 (2002).

14. T. L. Stewart and S. H. Ralston, "Role of genetic factors in the pathogenesis of osteoporosis," J. Endocrinol. 166(2), 235-245 (2000).

15. R. C. Pallotta et al., "Infrared (810-nm) low-level laser therapy on rat experimental knee inflammation," Lasers Med. Sci. 27(1), 71-78 (2012).
16. K. A. Samoilova et al., "Role of nitric oxide in the visible light-induced rapid increase of human skin microcirculation at the local and systemic levels: II. healthy volunteers," Photomed. Laser Surg. 26(5), 443-449 (2008).

17. C. H. Chen et al., "Low-level laser irradiation promotes cell proliferation and mRNA expression of type I collagen and decorin in porcine achilles tendon fibroblasts in vitro," J. Orthopaedic Res. 27(5), 646-650 (2009).

18. A. da Silva et al., "Effect of low-level laser therapy after rapid maxillary expansion on proliferation and differentiation of osteoblastic cells," Lasers Med. Sci. 27(4), 777-783 (2012).

19. P. S. Bossini et al., "Low level laser therapy $(830 \mathrm{~nm})$ improves bone repair in osteoporotic rats: similar outcomes at two different dosages," Exp. Gerontol. 47(2), 136-142 (2012).

20. Y. A. Vladimirov, A. N. Osipov, and G. I. Klebanov, "Photobiological principles of therapeutic applications of laser radiation," Biochemistry 69(1), 81-90 (2004).

21. P. A. de Castro et al., "Transcriptional profiling of Saccharomyces cerevisiae exposed to propolis," BMC Complementary Altern. Med. 12(1), 194 (2012).

22. Dana-Farber Cancer Institute, Boston, Massachusetts http://www.tm4. org/utilities.html.

23. Dana-Farber Cancer Institute, Boston, Massachusetts http://www.tm4. org/.

24. IPA®, QIAGEN Redwood City www.ingenuity.com.

25. K. C. Smith, "Laser (and LED) therapy is phototherapy," Photomed. Laser Ther. 23(1), 78-80 (2005).

26. K. D. Desmet et al., "Clinical and experimental applications of NIRLED photobiomodulation," Photomed. Laser Ther. 24(2), 121-128 (2006).

27. D. G. Minatel et al., "Phototherapy promotes healing of chronic diabetic leg ulcers that failed to respond to other therapies," Lasers Surg. Med. 41(6), 433-441 (2009).

28. K. R. Fernandes et al., "Effects of low-level laser therapy on the expression of osteogenic genes related in the initial stages of bone defects in rats," J. Biomed. Opt. 18(3), 038002 (2013).

29. T. O. F. de Souza et al., "Phototherapy with low-level laser affects the remodeling of types I and III collagen in skeletal muscle repair," Lasers Med. Sci. 26(6), 803-814 (2011).

30. G. M. Paraguassú et al., "Effect of laser phototherapy ( $\lambda 660 \mathrm{~nm})$ on type I and III collagen expression during wound healing in hypothyroid rats: an immunohistochemical study in a rodent model," Photomed. Laser Surg. 32(5), 281-288 (2014).

31. Y. S. Bland, M. A. Critchlow, and D. E. Ashhurst, "The expression of the fibrillar collagen genes during fracture healing: heterogeneity of the matrices and differentiation of the osteoprogenitor cells," Histochem. J. 31(12), 797-809 (1999).

32. F. Vázquez-Villa et al., "COL11A1/(pro) collagen 11A1 expression is a remarkable biomarker of human invasive carcinoma-associated stromal cells and carcinoma progression," Tumor Biol. 36(4), 2213-2222 (2015).

33. S. Takeda et al., "Hydrolyzed collagen intake increases bone mass of growing rats trained with running exercise," J. Int. Soc. Sports Nutr. 10(1), 35 (2013).

34. D. Saperia et al., "Demonstration of elevated type I and type III procollagen mRNA levels in cutaneous wounds treated with helium-neon laser: proposed mechanism for enhanced wound healing," Biochem. Biophys. Res. Commun. 138(3), 1123-1128 (1986).

35. C. C. S. Martignago et al., "Effect of low-level laser therapy on the gene expression of collagen and vascular endothelial growth factor in a culture of fibroblast cells in mice," Lasers Med. Sci. 30(1), 203-208 (2014).

36. F. Nudelman et al., "In vitro models of collagen biomineralization," J. Struct. Biol. 183(2), 258-269 (2013).

37. P. Fratzl et al., "Bone mineralization in an osteogenesis imperfecta mouse model studied by small-angle x-ray scattering," J. Clin. Invest. 97(2), 396-402 (1996).

38. A. N. Pereira et al., "Effect of low-power laser irradiation on cell growth and procollagen synthesis of cultured fibroblasts," Lasers Surgery Med. 31(4), 263-267 (2002).

39. L. S. Pugliese et al., "The influence of low-level laser therapy on biomodulation of collagen and elastic fibers," Pesquisa Odontológica Brasileira 17(4), 307-313 (2003). 
Tim et al.: Low-level laser therapy induces an upregulation of collagen gene expression...

40. M. J. Conlan, J. W. Rapley, and C. M. Cobb, "Biostimulation of wound healing by low-energy laser irradiation a review," J. Clin. Periodontol. 23(5), 492-496 (1996).

41. J. M. Bjordal, C. Couppe, and A. E. Ljunggren, "Low level laser therapy for tendinopathy. Evidence of a dose-response pattern," Phys. Ther. Rev. 6(2), 91-99 (2001).

42. P. M. Carrinho et al., "Comparative study using $685-\mathrm{nm}$ and $830-\mathrm{nm}$ lasers in the tissue repair of tenotomized tendons in the mouse," Photomed. Laser Ther. 24(6), 754-758 (2006).
43. G. Xiao et al., "Ascorbic acid-dependent activation of the osteocalcin promoter in MC3T3-E1 preosteoblasts: requirement for collagen matrix synthesis and the presence of an intact OSE2 sequence," Mol. Endocrinol. 11(8), 1103-1113 (1997).

Carla Roberta Tim is a postdoctorate researcher at the Federal University of São Paulo, Brazil.

Biographies for the other authors are not available. 